\title{
INVESTIGACIONES
}

\section{Clima Escolar, Autoconcepto académico y Calidad de Vida en alumnos/as de aulas culturalmente diversas*}

\author{
School Climate, Self-concept and Quality of Life \\ in students who attend culturally diverse classrooms
}

Sònia Lahoz i Ubach ${ }^{a}$

${ }^{a}$ Instituto Nacional de Derechos Humanos, Chile. slahoz@indh.cl

\begin{abstract}
RESUMEN
En este estudio se evalúa la dimensión social del Clima Escolar, la Calidad de Vida y el Autoconcepto Académico percibidos por estudiantes de educación básica y media, de escuelas y liceos de la Región Metropolitana escolarizados en aulas culturalmente diversas. La muestra fue conformada por 905 estudiantes (51,1\% hombres; 48,9\% mujeres) de nacionalidad chilena, venezolana, peruana y haitiana. Los resultados indican diferencias en la percepción del Clima Escolar según sexo y nacionalidad. La relación entre Clima Escolar, Autoconcepto Académico y la Calidad de Vida percibida, así como el valor explicativo del Clima Escolar y de la Percepción de Calidad de Vida en el Autoconcepto Académico también varían según la nacionalidad de las y los estudiantes. La dimensión de Calidad de Vida vinculada al Entorno Escolar, junto con el Apoyo al Pluralismo cultural y el Apoyo por parte de las y los profesores inciden en el Autoconcepto Escolar, explicando un $29 \%$ de su varianza. Los resultados suponen un insumo para el desarrollo de intervenciones en aulas multiculturales enfocadas a favorecer el Autoconcepto Académico del estudiantado de distintas nacionalidades. Se destaca la importancia de incluir el Apoyo al Pluralismo Cultural en la evaluación del Clima Escolar.
\end{abstract}

Palabras claves: Clima Escolar, estudiantes, Apoyo al Pluralismo Cultural, Autoconcepto Académico, inmigrantes.

\section{ABSTRACT}

The study explores the social dimension of the School Climate, and the association between School Climate and the Quality of Life and Academic Self-Concept perceived by students from $7^{\text {th }}$ grade to high school, who attend culturally diverse classrooms, public schools, in the city of Santiago. The sample was of 905 students $(51.1 \%$ men; $48.9 \%$ women) of Chilean, Venezuelan, Peruvian and Haitian nationality. The results identify differences by sex, and nationality in the perception of School Climate. The relationship between School Climate, Academic Selfconcept and perceived Quality of Life, and the explanatory value of School Climate and Perception of Quality of Life in Academic Self-concept show important differences according to the nationality of the students. The Quality of Life dimension associated to the School Environment, along with the Support for Cultural Pluralism and the Support from the teachers affect the School Self-Concept, explaining $29 \%$ of its variance. The results represent an input for the development of interventions in multicultural classrooms focused on promoting the Academic Selfconcept of students of different nationalities. The importance of including the Support for Cultural Pluralism in the evaluation of School Climate is highlighted.

Key words: School Social Climate, students, Support for Cultural Pluralism, Academic Self-Concept, immigrants.

\footnotetext{
* Esta investigación se ha realizado dentro del proyecto FONDECYT de Postdoctorado $N^{\circ} 3170528$ subvencionado por la
} Comisión Nacional de Investigación Científica y Tecnológica (CONICYT). 


\section{INTRODUCCIÓN}

En los últimos 30 años, Chile ha cobrado gran importancia en el sistema migratorio regional, convirtiéndose en un nuevo polo de recepción de migrantes tanto de Sudamérica como del Caribe. Este aumento de población migrante conlleva la presencia de niños, niñas y adolescentes extranjeros - o hijos de extranjeros - en las aulas, especialmente de escuelas municipales. Así lo demuestran los datos presentados en 2018 por el Ministerio de Educación (2018), que indican que entre 2015 y 2018 se habría casi cuadruplicado el número de estudiantes extranjeros/as en el sistema escolar chileno, de los cuales, el 57\% asiste a un establecimiento municipal. La Región Metropolitana cuenta con la mayor cantidad de estudiantes extranjeros/as, concentrando el 59,3\% del total. Este aumento de estudiantes extranjeros conlleva la aparición de un escenario multicultural en las aulas que hace necesario el desarrollo de estrategias que faciliten la convivencia entre las y los alumnos de los centros escolares.

El clima escolar es un elemento fundamental de la convivencia en la escuela (Mendoza \& Ballesteros, 2014). Sin embargo, hasta el día de hoy existe una falta de consenso sobre la definición de clima escolar y de los parámetros con los cuales se puede medir. Esto ha llevado a que el concepto "clima escolar" sea utilizado a menudo para abarcar muchos y diversos aspectos del entorno escolar (Claro, 2013; Thapa, Cohen, Guffey \& HigginsD’Alessandro, 2013; Wang \& Degol, 2016). A pesar de esta diversidad terminológica, se puede reconocer elementos comunes como es el reconocimiento que el clima se refiere a un fenómeno que se da en lo habitual/regular del transcurso de una comunidad escolar o en sub-grupos como puede ser el grupo-curso. Teniendo esto como base, algunas definiciones destacan que el clima es un fenómeno subjetivo, es decir una percepción de los miembros de la comunidad escolar, y otras un fenómeno objetivo, observado por un tercero (Claro, 2013).

Sobre la base que el clima es un fenómeno que emerge en las comunidades escolares, la literatura ha señalado la relación del clima escolar positivo, tanto con variables vinculadas a la percepción de calidad de vida por parte del alumnado -mejor bienestar subjetivo (Freeman et al., 2009; Konu, Lintonen, \& Autio, 2002; Varela et al., 2019, Ruus et al., 2007; Virtanen et al., 2009), mejor autoestima y autoconcepto (Beld et al., 2019; Steffgen, Rechhia \& Viechtbauer, 2013; Cornejo \& Redondo, 2001; Jia et al., 2009; Way, Reddy \& Rhodes, 2007) satisfacción vital (Leria \& Salgado, 2019), y satisfacción escolar (Zullig, Ward, Huebner \& Daily, 2017) - como con variables asociadas con los resultados académicos -promedio de calificaciones (Jia et al., 2009), autoconcepto académico (Coelho, Bear \& Brás, 2020; Ullman, 2014), mejores aptitudes matemáticas y verbales (Gietz \& McIntosh, 2014) y mejores resultados académicos (Berkowitz et al., 2015; Hanson \& Voight, 2014; McCoy, Roy \& Sirkman, 2013; OCDE, 2015; Persson \& Svensson, 2017)-. Además, se ha observado que un clima escolar negativo puede resultar en comportamientos disruptivos, deterioro de los logros de aprendizaje y aumento de agresiones entre estudiantes (Barth, Dunlap, Dane, Lochman \& Wells, 2004; Persson \& Svensson, 2017). Asimismo, un metaanálisis de 153 estudios encontró que el clima escolar era uno de los mejores predictores de acoso y victimización (Cook, Williams, Guerra, Kim \& Sadek, 2010).

Diferentes revisiones apuntan a la multidimensionalidad del clima escolar. Así, por ejemplo, el National School Climate Center (NSCC, 2012), identifica 12 dimensiones del clima escolar, agrupadas en 5 supradimensiones que serían: seguridad, enseñanza y 
aprendizaje, relaciones interpersonales, ambiente institucional y equipo de trabajo. Dentro de la supradimensión de relaciones interpersonales, el NSCC incluye el respeto por la diversidad, el apoyo social recibido de las y los adultas/as, el apoyo social recibido de las y los compañeros/as. De modo similar, Thapa et al. (2013) distinguen cinco dimensiones del clima escolar: seguridad, relaciones, enseñanza y aprendizaje, entorno institucional y proceso de mejora escolar. La dimensión de relaciones incluye apoyo social, respeto por la diversidad y conciencia de las diferencias raciales y étnicas. Igualmente, Wang y Degol (2016) reconocen cuatro dimensiones del clima escolar: académica, seguridad, institucional y comunitaria. Esta última dimensión abarcaría las relaciones entre la familia y la escuela, la calidad de las relaciones interpersonales dentro de la escuela, la vinculación de los y las estudiantes y el respeto por la diversidad.

Los estudios que abarcan la dimensión de comunidad o de relaciones han indicado que la percepción de relaciones interpersonales más positivas, tanto entre estudiantes como entre estudiantes y maestros/as, se asocia a una mayor satisfacción con la vida (Suldo, ThaljiRaitano, Hasemeyer, Gelley \& Hoy, 2013), mayor autoestima y mejores aprendizajes (Jia et al., 2009) y con niveles más bajos de participación en conductas problemáticas (Wang, Selman, Dishion \& Stormshak, 2010).

El respeto a la diversidad con frecuencia se destaca como variable crítica de la dimensión social del clima escolar (Brand, Felner, Shim, Seitsinger \& Dumas, 2003; NSCC, 2012; Thapa et al., 2013), sin embargo, su evaluación ha sido poco frecuente. Existen estudios que analizan las diferencias raciales en la percepción del clima escolar (p. ej. Bottiani, Bradshaw \& Mendelson, 2014, 2016; Fan, Williams \& Corkin, 2011; Konold, Cornell, Shukla \& Huang, 2017; Koth, Bradshaw \& Leaf, 2008; Voight, Hanson, O’Malley \& Adekanye, 2015), otros abordan clima escolar en contexto de migración (Pong \& Hao, 2007; Potochnick, Perreira \& Fuligni, 2012; Stone \& Han, 2005), y de forma más escasa, estudios sobre apoyo al pluralismo cultural como variable a considerar en el clima escolar.

Considerando las aulas culturalmente diversas, el apoyo al pluralismo cultural es cada vez más necesario. El pluralismo cultural se ha definido como la percepción que tienen las y los estudiantes del grado en que la diversidad cultural y la colaboración y la participación de las y los adolescentes pertenecientes a grupos de diferentes culturas son aspectos valorados y fomentados en la escuela (Brand et al., 2003). Los pocos estudios que incluyen esta dimensión en la evaluación del clima escolar han señalado que las y los estudiantes pertenecientes a grupos minoritarios, que perciben niveles más altos de apoyo al pluralismo cultural, muestran mayor adaptación socioemocional, expectativas y aspiraciones académicas más altas (Brand et al., 2003; Cristini, Scacchi, Perkins, Santinello \& Vieno, 2011; Le, Lai \& Wallen, 2009). Es destacable, sin embargo, que los estudios anteriores se han centrado en diferentes grupos raciales/étnicos_comparando la percepción del clima escolar según alumnos clasificados en las categorías de hispanos/latino, blancos, asiáticos y negros (Bottiani et al., 2016; Konold et al., 2017; Fan et al., 2011), pero no en las diferencias de percepción del clima escolar entre alumnos/as migrantes de diferentes nacionalidades y los nacionales del país de recepción de estos migrantes.

La investigación previa también ha establecido relaciones entre las percepciones de clima escolar y el sexo del estudiantado. Así, por ejemplo, Bottiani, Bradshaw y Mendelson (2016) en su estudio de casi 20.000 adolescentes de diferentes razas/etnias, encontraron que los estudiantes hombres reportaban niveles significativamente más altos de clima escolar. Alternativamente, Schneider y Duran (2010) hallaron que las alumnas mostraban 
significativamente una percepción más alta de Clima Escolar en un estudio de casi tres mil estudiantes de secundaria, mayoritariamente pertenecientes a grupos minoritarios. Otros estudios apoyan que las estudiantes mujeres valoran de forma más positiva el Clima Escolar (Fan et al., 2011; Koth et al., 2008; Guerra et al., 2012).

Por otro lado, la calidad de vida se ha asociado con el rendimiento académico (Buterin, 2019; Mok \& Flynn, 1997; Murray-Harvey, 2010). De forma específica, la calidad de vida vinculada al entorno escolar ha sido positivamente relacionada con un mejor rendimiento académico (Pietarinen, Soini \& Pyhältö, 2014).

Por todos estos elementos, el propósito del presente estudio fue explorar la dimensión social del clima escolar en el alumnado de distintas nacionalidades, que cursaban séptimo y octavo año de educación básica y de primero a cuarto año de educación media, escolarizados en aulas culturalmente diversas de la Región Metropolitana. Dada la investigación previa que demuestra las relaciones positivas entre el clima escolar y la calidad de vida percibida con el autoconcepto académico de los y las estudiantes, el segundo propósito fue determinar en qué medida las percepciones de los estudiantes sobre el Clima Escolar y la Calidad de Vida están asociadas con el Autoconcepto Académico y determinar cuáles serían las más importantes.

\section{MÉTODO}

\subsection{POBLACIÓN Y MUESTRA}

Participaron de la investigación un total de 19 establecimientos educacionales, todos pertenecientes al sistema de educación pública de la Región Metropolitana de Santiago y con presencia de alumnado migrante. La selección de la muestra fue de tipo intencional, y quedó conformada por 905 estudiantes (51,1\% hombres; 48,9\% mujeres) de séptimo y octavo año de educación básica $(37,7 \%)$ y de primero a cuarto año de educación media $(62,3 \%)$, con edades comprendidas entre los 11 y 19 años $(M=14,96 ; D E=1,81)$. Las escuelas y liceos seleccionados pertenecen al sistema de educación pública, de 10 comunas de la Región Metropolitana de Santiago, que cuentan con un porcentaje elevado de alumnos/ as de nacionalidades distinta a la chilena. Las nacionalidades de la muestra corresponden a: chilena $(67,8 \%)$, venezolana $(15 \%)$, peruana $(9,7 \%)$ y haitiana $(7,4 \%)$. El tiempo medio de estadía en Chile para cada una de estas nacionalidades era de 1,28 años para el alumnado venezolano, 2 para el haitiano y 5 para el peruano.

\subsection{MEDIDAS E INSTRUMENTOS}

Se midieron datos sociodemográficos de los y las alumnas (sexo, nacionalidad, y, en el caso de los y las alumnas extranjeras, tiempo de residencia en el país).

Se utilizaron, además, tres instrumentos para evaluar la calidad de vida subjetiva, el autoconcepto académico y la dimensión social del Clima Escolar.

El Kidsscreen-27 (Ravens-Sieberer et al., 2007) evalúa la calidad de vida subjetiva de niños/as y adolescentes de 8 a 18 años a través de 27 ítems distribuidos en 5 escalas: Bienestar Físico (5 ítems), que explora el nivel de actividad física, energía y estado físico del adolescente; Bienestar Psicológico (7 ítems), vinculada a las emociones positivas 
y satisfacción con la vida, así como la ausencia de sentimientos de soledad y tristeza; Autonomía y Relación con los padres (7 ítems), que recoge la calidad de la interacción entre el/la adolescente y sus padres/madres o cuidadores/as, atmósfera familiar, apoyado por la familia, nivel de autonomía percibido, y satisfacción con los recursos económicos; Apoyo Social y Pares (4 ítems) que explora la calidad de la interacción entre el/la adolescente con sus pares; Entorno Escolar (4 ítems) que explora la percepción de el/la adolescente de su capacidad cognitiva, aprendizaje y concentración y sus sentimientos acerca de la escuela. Los ítems de cada una de las escalas tienen un formato de respuesta tipo Likert de 5 puntos, variando desde 1 (nada o nunca) a 5 (muchísimo o siempre).

El Kidscreen-27 ha sido validado por Molina et al. (2014) en población adolescente chilena, de 10 a 18 años, obteniendo un alfa de Cronbach para el instrumento completo de .89 y puntuaciones mayores a .75 para todas las dimensiones, a excepción del dominio entorno escolar que obtuvo .69 .

Para el presente estudio la fiabilidad obtenida por el instrumento completo fue de 93. La Tabla 1 muestra los coeficientes de fiabilidad de cada una de las escalas, tanto los obtenidos por Molina et al. (2014) como los obtenidos en este estudio, para el total de la muestra y por nacionalidades.

Para evaluar el Autoncepto Académico se usó la escala homónima del cuestionario de Autoconcepto AF-5 (García \& Musitu, 2014) la cual evalúa, a través de seis ítems, la percepción que tiene el o la estudiante respecto a la calidad de su desempeño académico. En el contexto chileno, el cuestionario ha sido validado por Riquelme y Riquelme (2011). La Tabla 1 muestra los coeficientes de fiabilidad de esta escala, tanto los obtenidos por Riquelme y Riquelme (2011) como los obtenidos en este estudio, para el total de la muestra y por nacionalidades.

Finalmente, para evaluar la dimensión social del Clima Escolar, se utilizaron 4 subescalas del Inventory of School Climate-Student (ISC-S) de Brand et al. (2003): Apoyo del Profesorado, Interacciones Positivas entre Pares, Interacciones Negativas entre Pares, y Apoyo al Pluralismo Cultural. El ISC-S fue desarrollado para medir el clima escolar de estudiantes de $2^{\circ}$ grado de primaria. Los ítems de cada una de las escalas siguen un formato de respuesta tipo Likert de 5 puntos, variando desde 1 (nunca) a 5 (a menudo). No obstante las diferentes escalas que miden el Clima escolar hayan sido validadas en Chile, o son usadas por los organismos gubernamentales como la Agencia de Calidad de la Educación, dichas escalas no incluyen la dimensión de Apoyo al Pluralismo Cultural. Por esta razón, y dado que las escalas seleccionadas del ISC-S no habían sido utilizadas con anterioridad en el contexto chileno, fueron traducidas a partir del método de traducciónretrotraducción y, posteriormente a la adaptación madurativa e idiomática de los ítems, se realizó un análisis factorial confirmatorio (CFA) para determinar la validez y la estructura factorial de la dimensión social del Clima escolar, en un muestra de 1875 alumnos/as de diferentes nacionalidades (colombianos, chilenos, haitianos, peruanos y venezolanos). Los resultados del CFA proporcionaron un índice de ajuste suficiente (RMSEA= .04; $\mathrm{CFI}=.95$, $\mathrm{IFI}=.95, \mathrm{NFI}=.93, \mathrm{NNFI}=.94)$. La fiabilidad total de la escala, para el presente estudio, fue de .85. En la Tabla 1 se observan los coeficientes de fiabilidad de cada una de las escalas, tanto los obtenidos por Brand et al. (2003) como los obtenidos en este estudio, para el total de la muestra y por nacionalidades. 
Estudios Pedagógicos XLVII N 1: 7-25, 2021

CLIMA ESCOLAR, AUTOCONCEPTO ACADÉMICO Y CALIDAD DE VIDA EN ALUMNOS/AS DE AULAS CULTURALMENTE DIVERSAS

Tabla 1. Consistencia interna del cuestionario original y su variación entre nacionalidades de las y los participantes

\begin{tabular}{|c|c|c|c|c|c|c|c|}
\hline KIDSCREEN-27 & ítems & $\begin{array}{c}\alpha \\
\text { Molina et al. (2014) }\end{array}$ & $\begin{array}{c}\alpha \\
\text { muestra } \\
\text { total }\end{array}$ & $\begin{array}{c}\alpha \\
\text { Ch. }\end{array}$ & $\begin{array}{c}\alpha \\
\mathrm{H}\end{array}$ & $\begin{array}{l}\alpha \\
\mathrm{P} .\end{array}$ & $\begin{array}{l}\alpha \\
\mathrm{V}\end{array}$ \\
\hline Bienestar físico & 5 & .75 & .83 & .83 & .76 & .81 & .83 \\
\hline Bienestar psicológico & 7 & .81 & .83 & .84 & .60 & .84 & .84 \\
\hline $\begin{array}{l}\text { Autonomía y relación } \\
\text { con los padres }\end{array}$ & 7 & .83 & .83 & .84 & .79 & .84 & .78 \\
\hline Apoyo social y pares & 4 & .76 & .89 & .90 & .90 & .85 & .81 \\
\hline Entorno escolar & 4 & .69 & .75 & .77 & .28 & .72 & .68 \\
\hline Total & 27 & .89 & .93 & .93 & .92 & .91 & .92 \\
\hline AF-5 & ítems & $\begin{array}{c}\alpha \\
\text { Riquelme y Riquelme } \\
(2011)\end{array}$ & $\begin{array}{c}\alpha \\
\text { muestra } \\
\text { total }\end{array}$ & $\begin{array}{c}\alpha \\
\text { Ch. }\end{array}$ & $\begin{array}{c}\alpha \\
\mathrm{H}\end{array}$ & $\begin{array}{l}\alpha \\
\mathrm{P}\end{array}$ & $\begin{array}{l}\alpha \\
\mathrm{V}\end{array}$ \\
\hline Autoconcepto académico & 6 & .86 & .86 & .87 & .64 & .87 & .85 \\
\hline ISC-S & ítems & $\begin{array}{c}\alpha \\
\text { Brand et al. (2003) }\end{array}$ & $\begin{array}{c}\alpha \\
\text { muestra } \\
\text { total }\end{array}$ & $\begin{array}{c}\alpha \\
\text { Ch. }\end{array}$ & $\begin{array}{c}\alpha \\
\mathrm{H}\end{array}$ & $\begin{array}{l}\alpha \\
\mathrm{P}\end{array}$ & $\begin{array}{l}\alpha \\
\mathrm{V}\end{array}$ \\
\hline $\begin{array}{l}\text { Apoyo por parte de las y } \\
\text { los profesores }\end{array}$ & 6 & .76 & .85 & .86 & .79 & .83 & .83 \\
\hline $\begin{array}{l}\text { Interacciones negativas } \\
\text { entre compañeros/as }\end{array}$ & 5 & .79 & .80 & .78 & .72 & .82 & .83 \\
\hline $\begin{array}{l}\text { Interacciones positivas } \\
\text { entre compañeros/as }\end{array}$ & 5 & .70 & .84 & .85 & .72 & .78 & .82 \\
\hline $\begin{array}{l}\text { Apoyo al pluralismo } \\
\text { cultural }\end{array}$ & 5 & .68 & .69 & .69 & .70 & .72 & .61 \\
\hline $\begin{array}{l}\text { Dimensión Social del } \\
\text { Clima Escolar }\end{array}$ & 21 & - & .86 & .86 & .80 & .87 & .85 \\
\hline
\end{tabular}

Nota: Ch. = chilenos/as, $\mathrm{H} .=$ haitianos/as, $\mathrm{P} .=$ peruanos/as, $\mathrm{V} .=$ venezolanos/as

Se realizó un proceso de traducción-retrotraducción de los tres cuestionarios con el fin de desarrollar una versión de los mismos dirigida a las y los participantes de nacionalidad haitiana.

\subsection{PROCEDIMIENTO DE RECOGIDA Y ANÁLISIS DE DATOS}

Este estudio fue aprobado por el Comité de Ética de la Universidad Central de Chile. Los y las estudiantes que formaron parte de esta investigación participaron de manera voluntaria y anónima, con posterioridad a que sus padres, madres o apoderados firmaran el consentimiento y el/la adolescente el asentimiento. Se le informó, tanto a apoderados como 
a adolescentes, que la respuesta a los cuestionarios sería utilizada con fines exclusivamente académico-científicos y que la información facilitada era de carácter totalmente confidencial.

Los y las participantes respondieron los cuestionarios individualmente y de forma autónoma, en sesiones de 30-45 minutos, en el aula. El proceso de recogida de información se realizó entre los meses de marzo a diciembre de 2018. Este contempló el contacto con los directores de educación de las municipalidades de las comunas seleccionadas (aquellas que presentan mayor población migrante), solicitando su colaboración y, posteriormente, con la directiva de los colegios y liceos recomendados por los directores municipales, a quienes se les expuso el proyecto en sesiones informativas grupales e individuales. Con cada uno de los centros que confirmó su participación, se seleccionaron las aulas participantes, las fechas tentativas de administración de los cuestionarios y el procedimiento para facilitar y recoger la carta de consentimiento dirigida los apoderados. El proceso de administración de cuestionarios no comenzó hasta que el alumnado no hubiera estado en la escuela durante al menos 3 meses. En algunas oportunidades, dada la situación de tomas y marchas de los estudiantes, acontecidas en 2018, junto con niveles de ausentismo que no garantizaban la participación de más del $70 \%$ del estudiantado, las fechas de administración fueron postergadas. La administración de cuestionarios se efectuó entre julio y diciembre.

El análisis estadístico se realizó por medio del paquete estadístico SPSS (versión 25). En primer lugar, se examinó la distribución de los datos mediante la prueba de KolmogorovSmirnov. Dado que la mayoría de las variables no se ajustaron a una distribución normal, para el análisis de medias y correlaciones se utilizó estadística no paramétrica. Para la comparación de dos grupos se empleó la prueba de rangos de Wilcoxon y la prueba de Kruskal-Wallis, mientras que para la comparación de más de dos grupos se usó el test de Friedman y la U de Mann-Withney. Las correlaciones entre variables se evaluaron con la correlación de Spearman.

\section{RESULTADOS}

\subsection{PERCEPCIÓN DEL CLIMA ESCOLAR DEL ALUMNADO}

Para analizar si dentro de la muestra existían diferencias significativas entre las escalas evaluadas se ponderaron las puntuaciones de cada una de las escalas de 1 a 10 a fin de poder compararlas. El test de Friedman indicó que las puntuaciones de cada escala eran significativamente diferentes. Todos los pares comparados resultaron significativos mediante la prueba de rangos de Wilcoxon, siendo el Apoyo por parte de las y los profesores la dimensión que obtuvo significativamente mayor puntaje, seguida del Apoyo al pluralismo cultural. La dimensión Interacciones negativas entre compañeros/as es la que obtuvo menor puntuación (Tabla 2). 
Tabla 2. Puntuaciones medias y desviaciones estándar de las escalas de la dimensión social del Clima Escolar

\begin{tabular}{|l|c|c|c|c|}
\hline & $\begin{array}{c}\text { Apoyo al } \\
\text { pluralismo } \\
\text { cultural } \\
(5-20)\end{array}$ & $\begin{array}{c}\text { Apoyo por parte } \\
\text { de las y los } \\
\text { profesores } \\
(4-24)\end{array}$ & $\begin{array}{c}\text { Interacciones } \\
\text { negativas entre } \\
\text { compañeros/as } \\
(4-20)\end{array}$ & $\begin{array}{c}\text { Interacciones } \\
\text { positivas entre } \\
\text { compañeros/as } \\
(4-20)\end{array}$ \\
\hline Total muestra & $14.92(3.16)$ & $18.28(4.15)$ & $12.77(3.30)$ & $14.51(3.49)$ \\
\hline $\begin{array}{l}\text { Nacionalidad } \\
\text { chilena }\end{array}$ & $14.57(3.17)$ & $17.94(4.15)$ & $12.80(3.29)$ & $14.15(3.49)$ \\
\hline $\begin{array}{l}\text { Nacionalidad } \\
\text { extranjera }\end{array}$ & $15.70(3.00)$ & $19.01(3.69)$ & $12.69(3.67)$ & $15.32(3.19)$ \\
\hline $\begin{array}{l}\text { Nacionalidad } \\
\text { haitiana }\end{array}$ & $14.92(3.44)$ & $19.23(3.98)$ & $14.13(3.37)$ & $15.70(2.93)$ \\
\hline $\begin{array}{l}\text { Nacionalidad } \\
\text { peruana }\end{array}$ & $16.17(2.89)$ & $19.37(3.43)$ & $12.67(3.46)$ & $15.05(3.12)$ \\
\hline $\begin{array}{l}\text { Nacionalidad } \\
\text { venezolana }\end{array}$ & $15.71(2.83)$ & $18.69(3.74)$ & $12.08(3.78)$ & $15.33(3.34)$ \\
\hline Hombre & $14.64(3.14)$ & $17.96(4.12)$ & $12.61(3.51)$ & $14.30(3.43)$ \\
\hline Mujer & $15.25(3.14)$ & $18.62(3.94)$ & $12.97(3.30)$ & $14.72(3.44)$ \\
\hline
\end{tabular}

\subsection{PERCEPCIÓN DEL CLIMA ESCOLAR SEGÚN VARIABLES PERSONALES}

En la población extranjera se midió la relación entre tiempo en Chile y el Clima Escolar, la cual no fue significativa.

Se hallaron diferencias significativas al contrastar por sexo y nacionalidad de las y los estudiantes. Si bien al comparar entre alumnos y alumnas los resultados no fueron significativos en cuanto a la dimensión social del Clima Escolar, sí se observó que las mujeres puntúan, significativamente y de modo más favorable que sus pares hombres, el Apoyo al Pluralismo Cultural $(U=77760.50 ; p=.002)$ y el Apoyo por parte de las y los profesores $(U=79726.50 ; p=.020)$.

Al comparar entre las cuatro nacionalidades, se presentaron diferencias significativas entre todas ellas y en todas las dimensiones: Apoyo al Pluralismo Cultural ( $H$ de KruskalWallis $=29.39 ; p=.000)$, Apoyo por parte de las y los Profesores $(H$ de Kruskal-Wallis $=$ 13.04; $p=.005)$ e Interacciones Positivas entre Compañeros/as $(H$ de Kruskal-Wallis $=23.63$; $p=.000$ ) en las que el alumnado de nacionalidad chilena obtiene las menores puntuaciones, e Interacciones Negativas entre Compañeros/as ( $H$ de Kruskal-Wallis $=13.60 ; p=.004)$ la que es peor percibida por los y las alumnas de nacionalidad haitiana (Tabla 3). 
Tabla 3. Comparación de medias de Clima escolar entre nacionalidades

\begin{tabular}{|c|c|c|c|c|c|}
\hline & Chilenos/as & Haitianos/as & Peruanos/as & Venezolanos/as & $\mathrm{U}$ \\
\hline $\begin{array}{l}\text { Apoyo al } \\
\text { pluralismo } \\
\text { cultural }\end{array}$ & $14.57(3.17)$ & $14.92(3.44)$ & $16.17(2.89)$ & $15.71(2.83)$ & $\begin{array}{l}\mathrm{U}_{\text {ch.H. }}=14325.000 \\
\mathrm{U}_{\text {Ch.P. }}=17153.00^{* *} \\
\mathrm{U}_{\text {Ch.V. }}=28427.000^{* *} \\
\mathrm{U}_{\text {P.H. }}=1750.500^{*} \\
\mathrm{U}_{\text {P.V. }}=4645.500 \\
\mathrm{U}_{\text {H.V. }}=2890.500\end{array}$ \\
\hline $\begin{array}{l}\text { Apoyo por } \\
\text { parte de las y } \\
\text { los profesores }\end{array}$ & $17.94(4.15)$ & $19.23(3.98)$ & $19.37(3.43)$ & 18.69 (3.74) & $\begin{array}{l}\mathrm{U}_{\text {Ch.H. }}=12376.500^{*} \\
\mathrm{U}_{\text {Ch.P. }}=19073.000^{* *} \\
\mathrm{U}_{\text {Ch.V. }}=33924.000 \\
\mathrm{U}_{\text {P.H. }}=2096.000 \\
\mathrm{U}_{\text {P.V. }}=4778.000 \\
\mathrm{U}_{\text {H.V. }}=3018.000\end{array}$ \\
\hline $\begin{array}{l}\text { Interacciones } \\
\text { negativas entre } \\
\text { compañeros/as }\end{array}$ & $12.80(3.29)$ & $14.13(3.37)$ & $12.67(3.46)$ & 12.08 (3.78) & $\begin{array}{l}\mathrm{U}_{\text {ch.H. }}=11120.500 * * \\
\mathrm{U}_{\text {Ch.P. }}=22194.500 \\
\mathrm{U}_{\text {Ch.V. }}=30580.500 \\
\mathrm{U}_{\text {P.H. }}=1527.500 * \\
\mathrm{U}_{\text {P.V. }}=4312.500 \\
\mathrm{U}_{\text {H.V. }}=2136.500 * *\end{array}$ \\
\hline $\begin{array}{l}\text { Interacciones } \\
\text { positivas entre } \\
\text { compañeros/as }\end{array}$ & 14.15 (3.49) & $15.70(2.93)$ & $15.05(3.12)$ & 15.33 (3.34) & $\begin{array}{l}\mathrm{U}_{\text {ch.H. }}=10993.500^{* *} \\
\mathrm{U}_{\text {Ch.P. }}=18834.000 \\
\mathrm{U}_{\text {Ch.V. }}=29182.500^{* *} \\
\mathrm{U}_{\text {P.H. }}=1722.500 \\
\mathrm{U}_{\text {P.V. }}=4502.500 \\
\mathrm{U}_{\text {H.V. }}=3199.500\end{array}$ \\
\hline
\end{tabular}

Nota: $\mathrm{Ch}=$ chilenos/as; $\mathrm{H}=$ haitianos/as; $\mathrm{P}=$ peruanos/as; $\mathrm{V}=$ venezolanos/as.

* nivel de significación de $p<.05$

** nivel de significación de $p<.01$

\subsection{RELACIÓN ENTRE CLIMA ESCOLAR Y CALIDAD DE VIDA Y AUTOCONCEPTO ACADÉMICO}

Para analizar la relación entre la dimensión social del Clima Escolar sobre el Autoconcepto Académico y la Calidad de Vida se analizaron las correlaciones para toda la muestra y para cada una de las nacionalidades. En la Tabla 4 se pueden observar, para toda la muestra, correlaciones positivas medias entre la dimensión social del Clima Escolar con la Calidad de Vida $(r=.34 ; p=.000)$ y el Autoconcepto Académico $(r=.36 ; p=.000)$. Dichas correlaciones se mantienen en todas las nacionalidades, a excepción del alumnado de nacionalidad peruana, en el que no se observa correlación entre Clima Escolar y Calidad de Vida, y el alumnado venezolano en el que no se observan correlaciones entre las tres variables evaluadas. En el alumnado de nacionalidad haitiana los resultados muestran correlaciones positivas y fuertes entre Clima Escolar y el Autoconcepto Académico $(r=.67 ; p=.000)$. 
Tabla 4. Correlaciones entre la dimensión social del Clima Escolar,

Calidad de Vida, y Autoconcepto según nacionalidad de las y los participantes

\begin{tabular}{|l|l|c|c|}
\hline \multicolumn{1}{|c|}{} & Calidad de vida & Autoconcepto Académico \\
\hline \multirow{4}{*}{$\begin{array}{l}\text { Dimensión social del } \\
\text { Clima Escolar }\end{array}$} & Total muestra & $.34^{* *}$ & $.36^{* *}$ \\
\cline { 2 - 4 } & Chileno/as & $.34^{* *}$ & $.36^{* *}$ \\
\cline { 2 - 4 } & Haitianos & $.37^{*}$ & $.67^{* *}$ \\
\cline { 2 - 4 } & Peruanos &.- & $.28^{*}$ \\
\cline { 2 - 4 } & Venezolanos & $.37^{* *}$ &.- \\
\hline \multirow{3}{*}{ Calidad de vida } & Total muestra & & $.38^{* *}$ \\
\cline { 2 - 4 } & Chilenos & & $.39^{* *}$ \\
\cline { 2 - 4 } & Haitianos & & $.37^{*}$ \\
\cline { 2 - 4 } & Peruanos & & $.43^{* *}$ \\
\cline { 2 - 4 } & Venezolanos & &.- \\
\hline
\end{tabular}

* La correlación es significativa en el nivel .05 (bilateral)

** La correlación es significativa en el nivel .01 (bilateral)

Con el objetivo de profundizar en los resultados hallados, se efectuó un análisis correlacional entre las diferentes escalas que componen la dimensión social del Clima Escolar y la Calidad de Vida y el Autoconcepto Académico, para toda la muestra y para cada una de las nacionalidades. Como se observa en la Tabla 5 la escala de Calidad de Vida referida al Entorno Escolar correlaciona de manera media y fuerte con las escalas de Autoconcepto Académico, Apoyo al Pluralismo Cultural, Apoyo por parte del profesorado e Interacciones positivas entre compañeros/as. También se observan correlaciones medias entre el Autoconcepto Académico y las escalas de Clima Escolar, Apoyo al pluralismo cultural y Apoyo por parte del profesorado. 
Tabla 5. Correlaciones entre las escalas de la dimensión social del Clima Escolar y las escalas de Calidad de Vida, y el Autoconcepto Académico

\begin{tabular}{|l|c|c|c|c|c|}
\hline & $\begin{array}{c}\text { Autoconcepto } \\
\text { Académico }\end{array}$ & $\begin{array}{c}\text { Apoyo al } \\
\text { pluralismo } \\
\text { cultural }\end{array}$ & $\begin{array}{c}\text { Apoyo por } \\
\text { parte del } \\
\text { profesorado }\end{array}$ & $\begin{array}{c}\text { Interacciones } \\
\text { negativas }\end{array}$ & $\begin{array}{c}\text { Interacciones } \\
\text { positivas }\end{array}$ \\
\hline Autoconcepto Académico & - & $.33^{* *}$ & $.36^{* *}$ & - & $.29^{* *}$ \\
\hline Bienestar Físico & $.24^{* *}$ & $.18^{* *}$ & $.16^{* *}$ & - & $.18^{* *}$ \\
\hline Bienestar Psicológico & $.26^{* *}$ & $.15^{* *}$ & $.17^{* *}$ & $-.14^{* *}$ & $.23^{* *}$ \\
\hline Entorno escolar & $.47^{* *}$ & $.31^{* *}$ & $.35^{* *}$ & $-.12^{* *}$ & $.36^{* *}$ \\
\hline Autonomía & $.23^{* *}$ & $.13^{* *}$ & $.22^{* *}$ & $-.11^{* *}$ & $.19^{* *}$ \\
\hline Apoyo social & $.24^{* *}$ & $.18^{* *}$ & $.16^{* *}$ & $-.08^{*}$ & $.26^{* *}$ \\
\hline
\end{tabular}

* La correlación es significativa en el nivel .05 (bilateral)

** La correlación es significativa en el nivel .01 (bilateral)

\subsection{VALOR PREDICTIVO DEL CLIMA ESCOLAR Y LA CALIDAD DE VIDA EN EL AUTONCEPTO ACADÉMICO}

Finalmente, se realizaron análisis de regresión múltiple, con toda la muestra y con las submuestras por nacionalidad, para analizar la capacidad predictiva de las dimensiones sociales del Clima Escolar y la Calidad de Vida en relación al Autoconcepto Académico.

Tomada la muestra en su conjunto se observa (Tabla 6) que son significativas las dimensiones Entorno Escolar $(\beta=.42, \mathrm{t}=12.13, \mathrm{p}<.001)$, seguida del Apoyo al Pluralismo Cultural $(\beta=.13, t=3.27, p=.001)$, y el Apoyo por parte del profesorado $(\beta=-.11$, $t=2.68, p<.01)$. Estas dimensiones, en conjunto, explican el $29 \%$ de la varianza total del Autoconcepto Académico. Atendiendo a la cantidad de varianza explicada por cada dimensión, la Calidad de Vida vinculada al Entorno escolar, la primera en entrar explica el $25 \%$ del total, siendo significativa, pero escasa, la aportación de las dos dimensiones restantes (3.5\% Apoyo al Pluralismo Cultural y.70 \% el Apoyo por parte del profesorado).

Cuando se analiza la muestra separando por nacionalidades, se observa el mismo patrón anterior, para el alumnado chileno, siendo la dimensión Entorno escolar $(\beta=.46, \mathrm{t}=11.01$, $\mathrm{p}<.001)$, seguida del Apoyo al Pluralismo Cultural $(\beta=.12, t=2.40, p<.01)$, y el Apoyo por parte del profesorado $(\beta=.11, t=2.20, p<.01)$ las significativas, explicando el $32 \%$ de la varianza. Para el estudiantado haitiano, las dimensiones Apoyo al Pluralismo Cultural $(\beta=.34, t=2.50, p<.05)$, seguida por Interacciones Positivas entre Compañeros/as $(\beta=.50$, $t=3.59, \mathrm{p}<.005)$, llegan a explicar el $42 \%$ de la variación del Autoconcepto Académico. Para esta población, el Apoyo al Pluralismo Cultural explica el $34 \%$ del total, mientras que las Interacciones positivas entre compañeros/as da cuenta del $8 \%$ de la variación del Autoconcepto Académico. Finalmente, para el estudiantado peruano, las dimensiones de Entorno escolar y Apoyo al Pluralismo Cultural estarían explicando el $29 \%$ mientras que, en el alumnado venezolano, la dimensión Apoyo por parte del profesorado sería la única significativa, explicando solamente el $4 \%$ de la varianza. 


\begin{tabular}{|c|c|c|c|c|c|c|c|c|}
\hline \multicolumn{9}{|c|}{ Muestra total } \\
\hline $\mathbf{R}$ & $\mathbf{R}^{2}$ & $\begin{array}{c}\mathbf{R}^{2} \\
\text { corregida }\end{array}$ & $\begin{array}{c}\text { Error } \\
\text { estándar }\end{array}$ & $\mathbf{F}$ & Dimensiones Predictoras & $\boldsymbol{\beta}$ & $t$ & Sign. \\
\hline \multirow{3}{*}{.54} & \multirow{3}{*}{.29} & \multirow{3}{*}{.29} & \multirow{3}{*}{4.15} & \multirow{3}{*}{$95.64 * *$} & Entorno escolar & .42 & 12.13 & .000 \\
\hline & & & & & Apoyo al Pluralismo Cultural & .13 & 3.27 & .001 \\
\hline & & & & & Apoyo por parte del profesorado & .11 & 2.68 & .008 \\
\hline \multicolumn{9}{|c|}{ Chilenos/as } \\
\hline \multirow{3}{*}{.60} & \multirow{3}{*}{.32} & \multirow{3}{*}{.32} & \multirow{3}{*}{4.05} & \multirow{3}{*}{$76.30 * *$} & Entorno escolar & .46 & 11.01 & .000 \\
\hline & & & & & Apoyo al Pluralismo Cultural & .12 & 2.40 & .017 \\
\hline & & & & & Apoyo por parte del profesorado & .11 & 2.20 & .030 \\
\hline \multicolumn{9}{|c|}{ Haitianos/as } \\
\hline \multirow[b]{2}{*}{.67} & \multirow[b]{2}{*}{.45} & \multirow[b]{2}{*}{.42} & \multirow[b]{2}{*}{3.27} & \multirow[b]{2}{*}{$13.27 * *$} & Apoyo al Pluralismo Cultural & .34 & 2.50 & .018 \\
\hline & & & & & $\begin{array}{l}\text { Interacciones positivas entre } \\
\text { compañeros/as }\end{array}$ & .50 & 3.59 & .001 \\
\hline \multicolumn{9}{|c|}{ Peruanos/as } \\
\hline \multirow{2}{*}{.56} & \multirow{2}{*}{.31} & \multirow{2}{*}{.29} & \multirow{2}{*}{4.14} & \multirow{2}{*}{$15.31 * *$} & Entorno escolar & .47 & 4.71 & .000 \\
\hline & & & & & Apoyo al Pluralismo Cultural & .28 & 2.78 & .007 \\
\hline \multicolumn{9}{|c|}{ Venezolanos/as } \\
\hline .22 & .05 & .04 & 3.54 & $5.65^{*}$ & Apoyo por parte del profesorado & .22 & 2.38 & .019 \\
\hline
\end{tabular}

** La correlación es significativa en el nivel 0.01 (bilateral)

\section{DISCUSIÓN Y CONCLUSIONES}

El presente estudio tuvo como objetivo explorar la percepción del clima escolar en el alumnado de séptimo y octavo año de educación básica, y primero a cuarto año de educación media, de escuelas y liceos de la Región Metropolitana, escolarizados en aulas culturalmente diversas. El estudio examina las diferencias de percepción según sexo y nacionalidad de las y los estudiantes, además de las relaciones entre Clima Escolar, Calidad de Vida y Autoconcepto Académico de las y los estudiantes. Finalmente se evalúa el valor predictivo tanto del Clima Escolar como de la Calidad de Vida en el Autoconcepto Académico, analizándolo en función de su nacionalidad.

Respecto del primer objetivo, los resultados mostraron que las adolescentes valoran más positivamente que los hombres algunos aspectos del clima, como el Apoyo al Pluralismo Cultural y el Apoyo por parte de las y los Profesores, lo cual, además, es congruente con estudios previos que señalan la percepción más positiva del clima escolar por parte de las mujeres (Fan et al., 2011; Koth et al., 2008; Guerra et al., 2012).

La mejor percepción, a nivel general, se refiere al clima escolar vinculado al Apoyo por parte de las y los Profesores, mientras que la peor es la referida a las Interacciones Negativas entre Compañeros/as. 
Los resultados más interesantes de este estudio son los referidos a la percepción diferencial del clima escolar según la nacionalidad del alumnado. Si bien existen estudios que muestran que las y los estudiantes de diferentes grupos raciales/étnicos tienen percepciones diferentes del clima escolar - comparando la percepción del clima escolar según alumnos clasificados en las categorías de hispanos/latino, blancos, asiáticos y negros - (Bottiani et al. 2016; Konold et al., 2017), o que estudian el clima escolar en el contexto de la migración, y su papel moderador entre la discriminación y los síntomas depresivos (Cristini et al., 2011), existen pocas investigaciones que analicen las diferencias de percepción del clima escolar entre alumnos/as migrantes de diferentes nacionalidades y nacionales del país de recepción de estos migrantes. Estos estudios (Hombrados \& Castro, 2013; López, Barrós, Amigó \& Dubra, 2019) no reportan diferencias en la percepción del Clima Escolar, sin embargo, cabe señalar que estas investigaciones no han desglosado su análisis por nacionalidad, contemplando a las y los alumnos extranjeros bajo una misma categoría de "extranjeros" o "no nacionales". En este aspecto, el presente estudio significa un aporte al campo de investigación sobre clima escolar. Sus resultados muestran, no solo que el alumnado de diferentes nacionalidades percibe el clima de manera diferente, sino que el valor explicativo del clima escolar y de la calidad de vida en el autoconcepto académico es distinta dependiendo de la nacionalidad del alumnado. Así, en cuanto a las diferencias en la percepción del clima escolar, los alumnos y alumnas de nacionalidad chilena valoraron significativamente peor el clima referente al Apoyo al Pluralismo Cultural, Apoyo por parte de las y los Profesores e Interacciones Positivas entre Compañeros/as, mientras que las y los alumnos de nacionalidad haitiana perciben en mayor grado Interacciones Negativas entre Compañeros/as. El estudio da cuenta, asimismo, de que, a mayor percepción de un Clima Escolar positivo, mejor es el Autoconcepto Académico del alumnado, coincidiendo con investigaciones previas que ya habían señalado la relación entre ambas variables (Coelho, Bear \& Brás, 2020; Gietz \& McIntosh, 2014; Jia et al., 2009). Acorde con la literatura (Freeman et al., 2009; Konu et al., 2002; Varela et al., 2019), el estudio también da cuenta de la relación positiva ente Clima Escolar y Calidad de Vida. Sin embargo, estas relaciones positivas no se registran para todas las nacionalidades: para el alumnado peruano no se observa relación entre Clima Escolar y Calidad de Vida, mientras que, para el alumnado de nacionalidad venezolana, no se halla relación entre el clima y el Autoconcepto Académico.

El estudio encuentra también relación entre Calidad de Vida y Autoconcepto Académico para todos los estudiantes a excepción del estudiantado venezolano.

Si bien la presente investigación muestra asociación entre el clima escolar positivo y la calidad de vida de los y las estudiantes chilenos, es importante resaltar los resultados obtenidos en el alumnado migrante, principalmente considerando que el proceso migratorio puede cambiar la estructura familiar de los niños, niñas y adolescentes migrantes, generar contradicciones entre los valores familiares y los de la sociedad de llegada, y modificar la capacidad de los padres de prestar apoyo o dificultades para vincularse con sus pares debido a patrones diferentes de relación social (Hombrados \& Castro, 2013; Walsh, Shulman, Bar-On \& Tsur, 2006; Walsh, Harel-Fisch \& Fogel-Grinvald, 2010). Un clima escolar que propicie la percepción de mejor calidad de vida en las y los alumnos migrantes puede servir como protector ante las situaciones estresantes que niños, niñas y adolescentes migrantes deben afrontar en su proceso migratorio.

Los estudios realizados en Chile han señalado situaciones de discriminación del alumnado de nacionalidad haitiana en centros educativos, así como falta de integración 
en las redes de amistad con nacionales o migrantes de otras nacionalidades (Castillo, Santa-Cruz \& Vega, 2018; Pavel et al., 2018; Santa-Cruz, Thayer \& Troncoso, 2016). Situaciones de discriminación y dificultades de inclusión han sido, asimismo, reportadas por estudiantes de distinta nacionalidad (Hernández, 2016; Olate, 2018). Un clima escolar positivo, que propiciara las relaciones virtuosas entre profesores y alumnos/as, y entre estos últimos entre sí, así como la inclusión de la perspectiva cultural en las actividades del aula, podría convertirse en una fuente de apoyo que ayudara a niños, niñas y adolescentes migrantes a hacer frente a los cambios asociados a la migración (Cristini et al., 2011). Se ha señalado la importancia del clima multicultural en la salud mental y ajuste socioemocional: estudiantes extranjeros con una percepción positiva del clima multicultural en la escuela y el apoyo de los profesores presentan menos síntomas de depresión, mejor adaptación social y emocional y mejor ajuste escolar (Crosnoe, Johnson \& Elder, 2004; Le et al., 2009). La investigación reciente destaca una mayor influencia de las relaciones en la escuela sobre la salud mental de los estudiantes migrantes en comparación al apoyo de los padres (Walsh et al., 2010), y del apoyo social entre compañeros en la percepción de un clima escolar social más positivo, y en la disminución de los problemas de convivencia en el aula y de los conflictos debidos a las diferencias étnicas/culturales (Hombrados-Mendieta \& Castro-Travé, 2013). También se ha observado que el clima multicultural o el apoyo para el pluralismo cultural se asocia positivamente a niveles más bajos de discriminación (Bellmore, Nishina, You \& Ma 2012; Thijs \& Verkuyten, 2011).

Otros resultados del presente estudio indican la relación entre el Autoconcepto Académico y el Apoyo al pluralismo cultural y Apoyo por parte del profesorado -en cuanto a Clima escolar-, y el Entorno Escolar -en cuanto a Calidad de Vida-. Estos resultados van en la línea de las investigaciones que apuntan, en específico, la influencia del apoyo por parte de las y los profesores en el Autoconcepto del alumnado (Jia, et al., 2009). Paralelamente, los resultados del estudio también apoyan investigaciones previas que señalan la asociación entre el pluralismo cultural y el autoconcepto académico (Brand et al., 2003; Cristini et al., 2011; Le et al., 2009), y entre este y la calidad de vida vinculada al Entorno Escolar (Pietarinen, Soini \& Pyhältö, 2014).

Otro resultado destacable de esta investigación, a la hora de planificar estrategias de intervención, se refiere al efecto del Clima Escolar y de la calidad de vida vinculada al Entorno Escolar, en el Autoconcepto Académico, especialmente para estudiantes chilenos/ as y haitianos. El Autoconcepto académico ha sido utilizado por varias investigaciones como medida indirecta para el rendimiento académico real (Byrne \& Worth-Gavin, 1996; Kornilova, Kornilov \& Chumakova, 2009; Ullman, 2014), lo cual implicaría que, según los resultados obtenidos, la mejora del entorno escolar y del clima escolar a partir del Apoyo al Pluralismo Cultural, el Apoyo por parte del profesorado y las Interacciones positivas entre compañeros/as redundaría en la mejora del rendimiento académico del alumnado.

A nivel investigativo, los resultados del estudio también subrayan la importancia de incluir la evaluación, dentro del clima escolar, del apoyo al pluralismo cultural en el aula, más aún en las aulas culturalmente diversas.

Es necesario señalar la existencia de algunas limitaciones en la presente investigación. Por un lado, la baja confiabilidad que mostraron las sub-escalas de Entorno Escolar en la escala de Calidad de Vida, en el alumnado de nacionalidad haitiana, implica analizar las relaciones halladas entre esta variable y las sub-escalas de Clima Escolar con prudencia, específicamente para esta nacionalidad. Sin embargo, es importante resaltar que las 
escalas globales mostraron una buena consistencia interna, por lo que se puede concluir la existencia de una correlación efectiva entre la dimensión social del Clima Escolar, el Autoconcepto Académico y la Calidad de Vida.

Por otra parte, y debido a la naturaleza transversal de este estudio, los resultados deben interpretarse con cierta cautela, puesto que este tipo de trabajos no permiten establecer relaciones causa-efecto. Son necesarios estudios posteriores de corte longitudinal para poder confirmar el posible efecto causal.

Otra de las limitaciones tiene que ver con la ausencia de variables que pudiesen influir el Autoconcepto Académico para las y los alumnos peruanos y venezolanos. Es recomendable que futuras investigaciones incorporen nuevas dimensiones que ayuden a superar esta falencia.

Asimismo, sería recomendable realizar estudios con mayor participación de estudiantes migrantes a fin de poder confirmar las diferencias observadas en la percepción del clima escolar y en las relaciones entre esta y las otras variables estudiadas.

No obstante, y teniendo en cuenta las limitaciones señaladas, las aportaciones de este estudio son relevantes para al ámbito educativo al apuntar a la importancia de los centros educativos en influir y mejorar, a través del clima escolar y la calidad de vida en el autoconcepto académico de sus alumnos/as. El proporcionar diversas y diferenciales formas de apoyo social es un beneficio para los niños, niñas y adolescentes tanto migrantes como de la sociedad de llegada: apoyo social de las y los maestros, de las y los compañeros de curso y apoyo a la pluralidad cultural.

\section{REFERENCIAS BIBLIOGRÁFICAS}

Barth, J. M., Dunlap, S. T., Dane, H., Lochman, J. E. \& Wells, K. C. (2004). Classroom environment influences on aggression, peer relations and academic focus. Journal of school psychology 42(2), 115-133. doi: 10.1016/j.jsp.2003.11.004

Bear, G. G., Yang, C., Mantz, L. S. \& Harris, A. B. (2017). Schoolwide practices associated with school climate in elementary, middle, and high schools. Teacher and Teacher Education 63, 372-383. doi: 10.1016/j.tate.2017.01.012

Beld, M. H. M., Kuiper, C. H. Z., Van Der Helm, G. H. P., De Swart, J. J. W., Stams G. J. J. M. \& Roest J. J. (2019). Classroom Climate, Identification with School, and General Self-worth Predict Academic Self-Concept in Students Attending Residential Schools for Special Education. Residential Treatment for Children \& Youth, 1-16. doi: 10.1080/0886571X.2019.1696262

Bellmore, A., Nishina, A., You, J. y Ma, T. (2012). School context protective factors against peer ethnic discrimination across the high school years. American Journal of Community Psychology 49, 98-111. doi: 10.1007/s10464-011-9443-0

Berkowitz, R., Glickman, H., Benbenishty, R., Ben-Artzi, E., Raz, T., Lipshtadt, N. \& Astor, R. A. (2015). Compensating, mediating, and moderating effects of school climate on academic achievement gaps in Israel. Teachers College Record, 117, 1-34.

Berman, N. G. \& Parker, R. A. (2002). Meta-analysis: Neither quick nor easy. BMC medical research methodology 2(10). doi: 10.1186/1471-2288-2-10

Bottiani, J. H., Bradshaw, C. P. \& Mendelson, T. (2016). Inequality in Black and White high school students' perceptions of school support: An examination of race in context. Journal of Youth and Adolescence 45, 1176-1191. doi: 10.1007/s10964-015-0411-0

Bottiani, J. H., Bradshaw, C. P. \& Mendelson, T. (2014). Promoting an equitable and supportive school climate in high schools: The role of school organizational health and staff burnout. Journal of School Psychology 52(6), 567-582. doi: 10.1016/j.jsp.2014.09.003 
Brand, S., Felner, R., Shim, M., Seitsinger, A. \& Dumas, T. (2003). Middle school improvement and reform: Development and validation of a school-level assessment of climate, cultural pluralism, and school safety. Journal of educational psychology 95(3), 570. doi: 10.1037/00220663.95.3.570

Buterin, M. (2019). Quality of School Life in Primary School: Students' Perception. Pedagogika 134, 135-150. doi: 10.15823/p.2019.134.9.

Byrne, B. M. \& Gavin, D. A. W. (1996). The Shavelson Model revisited: Testing for the structure of academic self-concept across pre-, early, and late adolescents. Journal of Educational Psychology, 88(2), 215-228. doi: 10.1037/0022-0663.88.2.215

Castillo, D., Santa-Cruz, E. \& Vega, A. (2018). Estudiantes migrantes en escuelas públicas chilenas. Calidad en la educación, (49), 18-49.

Claro, J. S. (2013). Calidad en educación y clima escolar: apuntes generales. Estudios pedagógicos (Valdivia) 39(1), 347-359. doi: 10.4067/S0718-07052013000100020

Coelho, V. A., Bear, G. G. \& Brás, P. (2020). A multilevel analysis of the importance of school climate for the trajectories of students' self-concept and self-esteem throughout the middle school transition. Journal of youth and adolescence 49(9), 1793-1804. doi: 10.1007/s10964020-01245-7

Cook, C. R., Williams, K. R., Guerra, N. G., Kim, T. E. \& Sadek, S. (2010). Predictors of bullying and victimization in childhood and adolescence: A meta-analytic investigation. School Psychology Quarterly, (25), 65-83. doi: 10.1037/a0020149

Cornejo, R. \& Redondo, J. M. (2001). El clima escolar percibido por los alumnos de enseñanza media: Una investigación en algunos liceos de la Región Metropolitana. Última década 9(15), 11-52.

Cristini, F., Scacchi, L., Perkins, D. D., Santinello, M. \& Vieno, A. (2011). The influence of discrimination on immigrant adolescents' depressive symptoms: What buffers its detrimental effects? Psychosocial Intervention 20(3), 243-253. doi: 10.5093/in2011v20n3a2

Crosnoe, R., Johnson, M. K. \& Elder Jr, G. H. (2004). Intergenerational bonding in school: The behavioral and contextual correlates of student-teacher relationships. Sociology of education, 77(1), 60-81. doi:10.1177/003804070407700103

Fan, W., Williams, C. M. \& Corkin, D. M. (2011). A multilevel analysis of student perceptions of school climate: The effect of social and academic risk factors. Psychology in the Schools 48, 632-647. doi: 10.1002/pits.20579

Freeman, J. G., Samdal, O., Klinger, D. A., Dur, W., Griebler, R., Currie, D. \& Rasmussen, M. (2009). The relationship of schools to emotional health and bullying. International journal of public health 54(2), 251-259. doi: 10.1007/s00038-009-5421-9

García, F. \& Musitu, G. (2014). Autoconcepto forma 5. Madrid: Tea.

Gietz, C., \& McIntosh, K. (2014). Relations between student perceptions of their school environment and academic achievement. Canadian Journal of School Psychology, 29, 161-176. doi: $10.1177 / 0829573514540415$

Guerra, C., Vargas, J., Castro, L., Plaza, H. \& Barrera, P. (2012). Percepción del clima escolar en estudiantes de enseñanza media de Valparaíso de colegios municipales, particulares subvencionados y particulares. Estudios pedagógicos (Valdivia) 38(2), 103-115. doi: 0.4067/ S0718-07052012000200007

Hanson, T. \& Voight, A. (2014). The appropriateness of a California student and staff survey for measuring middle school climate. Washington, DC: U.S. Department of Education, Institute for Education Sciences, National Center for Education Evaluation and Regional Assistance, Regional Educational Laboratory West.

Hernández, A. (2016). El currículo en contextos de estudiantes migrantes: Las complejidades del desarrollo curricular desde la perspectiva de los docentes de aula. Estudios pedagógicos (Valdivia) 42(2), 151-169. doi: 10.4067/S0718-07052016000200009 
Hombrados-Mendieta, I. \& Castro-Travé, M. (2013). Apoyo social, clima social y percepción de conflictos en un contexto educativo intercultural. Anales de Psicología/Annals of Psychology 29(1), 108-122. doi: 10.6018/analesps.29.1.123311

Jia, Y., Way, N., Ling, G., Yoshikawa, H., Chen, X., Hughes, D., ... \& Lu, Z. (2009). The influence of student perceptions of school climate on socioemotional and academic adjustment: A comparison of Chinese and American adolescents. Child development 80(5), 1514-1530. doi: 10.1111/j.1467-8624.2009.01348.x

Konold, T., Cornell, D., Shukla, K. \& Huang, F. (2017). Racial/ethnic differences in perceptions of school climate and its association with student engagement and peer aggression. Journal of youth and adolescence 46(6), 1289-1303. doi: 10.1007/s10964-016-0576-1

Konu, A. I., Lintonen, T. P. \& Autio, V. J. (2002). Evaluation of Well-Being in Schools? A Multilevel Analysis of General Subjective Well-Being. School Effectiveness and School Improvement 13(2), 187-200. doi: 10.1076/sesi.13.2.187.3432

Kornilova, T. V., Kornilov, S. A. \& Chumakova, M. A. (2009). Subjective evaluations of intelligence and academic self-concept predict academic achievement: Evidence from a selective student population. Learning and Individual Differences 19(4), 596-608. doi: 10.1016/j.lindif.2009.08.001

Koth, C. W., Bradshaw, C. P. \& Leaf, P. J. (2008). A multilevel study of predictors of student perceptions of school climate: The effect of classroom-level factors. Journal of educational psychology 100(1), 96-104. doi: 10.1037/0022-0663.100.1.96

Le, T. N., Lai, M. H. \& Wallen, J. (2009). Multiculturalism and subjective happiness as mediated by cultural and relational variables. Cultural Diversity and Ethnic Minority Psychology 15(3), 30313. doi: $10.1037 / \mathrm{a} 0015507$

Leria-Dulčić, F. J. \& Salgado-Roa, J. A. (2019). Efecto del clima social escolar en la satisfacción con la vida en estudiantes de primaria y secundaria. Revista Educación 43(1), 364-379. doi: 10.15517/REVEDU.V43I1.30019

López, S., Barrós, C., Amigó, M. F. \& Dubra, M. (2019). Immigrant students in Spain compared to Spanish students: perceived relationships and academic results. Revista iberoamericana de psicología y salud 10(2), 64-74.

McCoy, D. C., Roy, A. L. \& Sirkman, G. M. (2013). Neighborhood crime and school climate as predictors of elementary school academic quality: A cross-lagged panel analysis. American Journal of Community Psychology 52(1-2), 128-140. doi: 10.1007/s10464-013-9583-5.

Mendoza, K. H. \& Ballesteros, R. R. (2014). El clima escolar como elemento fundamental de la convivencia en la escuela. Escenarios 12(2), 7-18. doi: 10.15665/esc.v12i2.311

Ministerio de Educación, Centro de Estudios (2018). Lanzamiento de la Política Nacional de Estudiantes Extranjeros, 2018-2022. Última visita 31 de agosto de 2019. Recuperado desde https://centroestudios.mineduc.cl/wp-content/uploads/sites/100/2018/05/PRESENTACION_ FINAL_POLITICA_EXTRANJEROS-1.pdf

Mok, M. \& Flynn, M. (1997). Quality of school life and students' achievement in the HSC: A multilevel analysis. Australian Journal of Education 41(2), 169-188.

Molina, T., Montaño, R., González, E., Sepúlveda, R., Hidalgo-Rasmussen, C., Martínez, V., ... \& George, M. (2014). Propiedades psicométricas del cuestionario de calidad de vida relacionada con la salud KIDSCREEN-27 en adolescentes chilenos. Revista Médica de Chile 142(11), 14151421. doi: $10.4067 /$ S0034-98872014001100008

Murray-Harvey, R. (2010). Relationship influences on students' academic achievement, psychological health and well-being at school. Educational and Child Psychology 27(1), 108-119.

National School Climate Center [NSCC]. (2012). The 12 dimensions of school climate measured. Recuperado de https ://www.schoolclimate.org

O’Malley, M., Voight, Bottiani, J. H., Bradshaw, C. P. \& Mendelson, T. (2014). Promoting an equitable and supportive school climate in high schools: The role of school organizational health and staff burnout. Journal of School Psychology 52(6), 567-582. doi: 10.1016/j.jsp.2014.09.003. 
OCDE. (2015). La comparación internacional para la mejora escolar. Madrid, España: OCDE. Última visita 12 de junio de 2019. Recuperado desde https://www.oecd.org/pisa/aboutpisa/Brochure.pdf

Olate, M. L. M. (2018). Política educativa para migrantes en Chile: un silencio elocuente. Polis. Revista Latinoamericana, (49). doi: 10.4067/S0718-65682018000100231

Pavez-Soto, I., Ortiz-López, J. E., Jara, P., Olguín, C. \& Domaica, A. (2018). Infancia haitiana migrante en Chile: barreras y oportunidades en el proceso de escolarización. EntreDiversidades, (11), 71-97. doi: 10.31644/ED.11.2018.a03

Persson, L., Svensson, M. (2017). Classmate characteristics, class composition and children's perceived classroom climate. Journal of Public health 25, 473-480. doi: 10.1007/s10389-017-0809-0

Pietarinen, J., Soini, T. \& Pyhältö, K. (2014). Students' emotional and cognitive engagement as the determinants of well-being and achievement in school. International Journal of Educational Research 67, 40-51.

Pong, S. L. \& Hao, L. (2007). Neighborhood and school factors in the school performance of immigrants' children. International Migration Review 41(1), 206-241.

Potochnick, S., Perreira, K. M. \& Fuligni, A. (2012). Fitting in: The roles of social acceptance and discrimination in shaping the daily psychological well-being of Latino youth. Social Science Quarterly 93(1), 173-190.

Ravens-Sieberer, U., Auquier, P., Erhart, M., Gosch, A., Rajmil, L., Bruil, J., ... \& Mazur, J. (2007). The KIDSCREEN-27 quality of life measure for children and adolescents: psychometric results from a cross-cultural survey in 13 European countries. Quality of Life Research 16(8), 13471356. doi: 10.1007/s11136-007-9240-2

Riquelme, E. \& Riquelme, P. (2011). Análisis Psicométrico confirmatorio de la medida multidimensional del Test de Autoconcepto Forma 5 en Español (AF5), en estudiantes Universitarios de Chile. Psicologia, saúde \& doenças 12(1), 91-103.

Ruus, V. R., Veisson, M., Leino, M., Ots, L., Pallas, L., Sarv, E. S. \& Veisson, A. (2007). Students' well-being, coping, academic success, and school climate. Social Behavior and Personality: An International Journal 35, 919-936. doi: 10.2224/sbp.2007.35.7.919

Santa-Cruz, E., Thayer, E. \& Troncoso, A. (2016). Inclusión y procesos de escolarización en estudiantes migrantes que asisten a establecimientos de educación básica. Última visita 15 de agosto de 2019. Recuperado desde https://centroestudios.mineduc.cl/wp-content/uploads/ sites/100/2017/07/INFORME-FINAL-F911463.pdf

Schneider, S. H. \& Duran, L. (2010). School climate in middle schools: A cultural perspective. Journal of Research in Character Education 8(2), 25-37.

Simpson, E. H. (1949). Measurement of diversity. Nature 163(4148), 688-688. doi: 10.1038/163688a0

Steffgen, G., Rechhia, S. \& Viechtbauer, W. (2013). The link between school climate and violence in school: A meta-analytic review. Aggression and Violent Behavior, 18, 300-309. doi:10.1016/j. avb.2012.12.001

Stone, S. \& Han, M. (2005). Perceived school environments, perceived discrimination, and school performance among children of Mexican immigrants. Children and Youth Services Review 27, 51-66. doi: 10.1016/j.childyouth.2004.08.011

Suldo, S. M., Thalji-Raitano, A., Hasemeyer, M., Gelley, C. D. \& Hoy, B. (2013). Understanding middle school students' life satisfaction: does school climate matter? Applied Research in Quality of Life 8, 169-182. doi: 10.1007/s11482-012-9185-7

Thapa, A., Cohen, J., Guffey, S. \& Higgins-D'Alessandro, A. (2013). A review of school climate research. Review of Educational Research 83, 357-385. doi: 10.3102/0034654313483907

Thijs, J. y Verkuyten, M. (2011). In-group bias in the classroom: The role of co-ethnic and otherethnic peers and multiculturalism. Anales de Psicología 27(3), 662-669.

Ullman, J. (2014). 'At-risk' or school-based risk? Testing a model of school-based stressors, coping responses, and academic self-concept for same-sex attracted youth. Journal of Youth Studies 18(4), 417-4337. doi: 10.1080/13676261.2014.963539. 
UNESCO; Idea Chile. (2005). Primer estudio nacional de convivencia escolar: La opinión de estudiantes y docentes.

Varela, J. J., Sirlopú, D., Melipillán, R., Espelage, D., Green, J. \& Guzmán, J. (2019). Exploring the Influence School Climate on the Relationship between School Violence and Adolescent Subjective Well-Being. Child Indicators Research 12(6), 2095-2110. doi: 10.1007/s12187-01909631-9

Virtanen, M., Kivimaki, M., Luopa, P., Vahtera, J., Elovainio, M., Jokela, J. \& Pietikainen, M. (2009). Staff reports of psychosocial climate at school and adolescents' health, truancy and health education in Finland. European Journal of Public Health 19, 554-560. doi: 10.1093/eurpub/ ckp032

Voight, A., Hanson, T., O’Malley, M. \& Adekanye, L. (2015). The racial school climate gap: Withinschool disparities in students' experiences of safety, support, and connectedness. American journal of community psychology 56(3-4), 252-267. doi: 10.1007/s10464-015-9751-x

Walsh, S. D., Harel-Fisch, Y. \& Fogel-Grinvald, H. (2010). Parents, teachers and peer relations as predictors of risk behaviors and mental well-being among immigrant and Israeli born adolescents. Social science \& medicine 70(7), 976-984. doi: 10.1016/j.socscimed.2009.12.010

Walsh, S., Shulman, S., Bar-On, Z. \& Tsur, A. (2006). The role of parentification and family climate in adaptation among immigrant adolescents in Israel. Journal of Research on Adolescence 16, 321-349. doi: 10.1111/j.1532-7795.2006.00134.x

Wang, M. T. \& Degol, J. L. (2016). School climate: A review of the construct, measurement, and impact on student outcomes. Educational Psychology Review 28(2), 315-352. doi: 10.1007/ s10648-015-9319-1

Wang, M. T., Selman, R. L., Dishion, T. J. \& Stormshak, E. A. (2010). A Tobit regression analysis of the covariation between middle school students' perceived school climate and behavioral problems. Journal of Research on adolescence 20(2), 274-286. doi: 10.1111/j.15327795.2010.00648.x

Way, N., Reddy, R. \& Rhodes, J. (2007). Students' perceptions of school climate during the middle school years: Associations with trajectories of psychological and behavioral adjustment. American journal of community psychology 40(3-4), 194-213. doi: 10.1007/s10464-007-9143-y

Zullig, K. J., Ward, R. M., Huebner, E. S. y Daily, S. M. (2017). Association between Adolescent School Climate and Perceived Quality of Life. Child Ind Res 11(6), 1737-1753. doi:10.1007/ s12187-017-9521-4 
